\title{
Entropy of Topological Space S and Evolution of Phase Space of Dynzmical Systems
}

\author{
Malkhaz Mumladze \\ Department of Education, Exact and Natural Sciences, Gori University, Gori, Georgia \\ Email address: \\ malkhazmumladze@gmail.com

\section{To cite this article:} \\ Malkhaz Mumladze. Entropy of Topological Space S and Evolution of Phase Space of Dynzmical Systems. International Journal of \\ Management and Fuzzy Systems. Vol. 6, No. 1, 2020, pp. 8-13. doi: 10.11648/j.ijmfs.20200601.12
}

Received: April 26, 2020; Accepted: June 1, 2020; Published: June 15, 2020

\begin{abstract}
In this paper, we introduced the concept of pseudo-convex open covering of topological spaces and entropy for topological spaces with such covering. Entropy was previously defined only for open coverings of compact topological spaces. It is shown by examples that the classes of topological spaces for which the concept of entropy is defined is quite wide. A discrete random process describing the evolution of the phase space of closed dynamical systems is built. Two random processes are constructed, one in which the elements of the transition matrices depend on the first indices, and the second Markov`s process, one in which the elements of the transition matrices do not depend from the first indices. The construction of transition matrices is based on the fact that the probability of a change in the phase space of a system to another space is proportional to the entropy of this other space. Based on the concept of entropy of topological spaces and on the well-known construction of an infinite product of probability measures which is also probabilistic introduced the concept entropy of trajectory of evolution of phase space of system. Previously, the entropy of the trajectory was defined only for the motion of a structureless material point, in this article is defined entropy of trajectory of evolution of structured objects represented in the form of topological spaces. On the basis of the concept entropy of trajectory, a method is determined for finding the most probable trajectory of evolution of the phase space of a closed system.
\end{abstract}

Keywords: Topological Space, Covering, Entropy, Random Process

\section{Introduction}

The evolution of real dynamical systems is reflected in the change intheir entropy. This fact must be taken into account in the mathematical modeling of such systems, so it is important to define the entropy concepts of the mathematical objects in which the dynamic systems are represented in the models. Often modeling of a dynamical systems (it's phase spaces) is considered to be a continuous or discrete sequence of topological spaces that describe the continuous or discrete time variability of the system. This sequence we called trajectory of changes of phase space. The notion of entropy of topological spaces has not been defined until recently. Until now, has been defined the concept of the entropy of covering of compact topological spaces $[8,9]$. In this work we have tried to define entropy directly for topological spaces and define entropy of trajectory.

This notion of entropy of a trajectory differs from other similar definitions. In other definitions, the trajectory is considered as trace of the movement of an unstructured point in the phase space $[8-10,15]$ and here as the trajectory of the changes of structured object, phase space of the system.

\section{Entropy for Topological Space}

Definition1. We called a pseudo-convex open covering of space $X$ the covering containing only contractible [5] elements, such sets whose intersections with a finite number are also contractible.

Definition 2. Call the entropy of a topological space $X$ number $\frac{n}{m}$ where $n$ is minimal number of subsets of open pseudo- convex coverings among finite coverings of $X$ and $m$ is the number of orbits in every $n$ element-containing coverings at the actions bay covering preserving corresponding homomorphisms. Denote the entropy of topological space $X$ so: $H(X)$. It is ease that the numbers applied in definition entropy $n$ and $m$ are in the relation: 
$n \geq m$.

There exist topological spaces which have pseudo-convex covering. For example:

1) The $n$ dimensional sphere.

2) Open subset in Tikhonov`s cube, Indeed, we consider the Tikhonov`s cube $I^{A}[14]$ where $A$ such set $\operatorname{card} A$ infinite cardinal number. If $x, y \in I=[0,1]$ and $\lambda \in I$ then $\lambda x+(1-\lambda) y \in I \quad, \quad$ it follows: if $\left\{x_{v}\right\},\left\{y_{v}\right\} \in I^{A}$ then $\lambda\left\{x_{\alpha}\right\}+(1-\lambda)\left\{y_{\alpha}\right\} \in I^{A}$ for every $\lambda \in I$ this means that in the cube we have the notion of a convex [4] set. The family of all open convex sets $\left\{O_{\alpha}\right\}$ in $I^{A}$ is base of Tikhonov topology since this family convex in $I^{A}$ and intersection of two open convex sets is convex. Let $X$ the subspace in $I^{A}$, the base in this subspace is family $\left\{O_{\alpha} \cap X\right\}$. If $X$ is open set then there is base of subspace $X$ which contains only convex sets.

3) Subsets of Tikhonov`s cube which are union of convex subsets.

4) For every absolute neighborhood retracts (ANR) $X$ there exist space $E$ that $X \times E$ has a pseudo convex base [1]

5) Let subspace $X \subset I^{\aleph_{0}}$ in Hilbert cube $I^{\aleph_{0}}$ [14] is $Q$ manifold [1]. If this manifold is open subset then it has a open pseudo-convex covering so above, for open set in Tikhonov`s cube. If the $X Q$ - manifold is closed set in $I^{\aleph_{0}}$ then set $X \times[0,1) \subset I^{\aleph_{0}}$ open in $I^{\aleph_{0}}$ [1]. This means that this space has open pseudo- convex covering. The space $X \times[0,1) \subset I^{\aleph_{0}}$ homotopy equivalent [5] to $X$. Every convex set is contractible. The space $X$ is deformation retract [5] in $X \times[0,1) \subset I^{\aleph_{0}}$ it follows that

$X \stackrel{i}{\longrightarrow} X \times[0,1) \stackrel{r}{\longrightarrow} X, r \circ i=I d_{a}, X \times[0,1) \stackrel{r}{\longrightarrow} X \stackrel{i}{\longrightarrow} X \times$

If $\left\{O_{\alpha}^{X \times[0,1)}\right\}$ is the basis consisting bay convex sets in $X \times[0,1) \subset I^{\aleph_{0}}$. then $\left\{i^{-1}\left(O_{\alpha}^{X \times[0,1)}\right\}\right.$ is open covering of $X$. Consider $i^{-1}\left(O_{\alpha}^{X \times[0,1)}\right)$, this set is contractible in $I^{\aleph_{0}}$. Indeed, let it is not so, then $r^{-1}\left(i^{-1}\left(O_{\alpha_{2}}^{X \times[0,1)}\right)\right)$ is not contractible in $X \times[0,1)$, since $r$ deformation retraction [5]. The set $(i \circ r)\left(r^{-1}\left(i^{-1}\left(O_{\alpha}^{X \times[0,1)}\right)\right)=O_{\alpha}^{X \times[0,1)}\right.$ can will be contracted to $I d_{X}\left(r^{-1}\left(i^{-1}\left(O_{\alpha_{2}}^{X \times[0,1)}\right)\right)\right.$ which is not contractible. So we got a contradiction non contractible set homotopy equivalent to contractible. It follows that our assumption is wrong. Because for open convex sets from $\left\{O_{\alpha}^{X \times[0,1)}\right\}$, the set $i^{-1}\left(O_{\alpha}^{X \times[0,1)}\right)$ is contractible, the family $\left\{i^{-1}\left(O_{\alpha}^{X \times[0,1)}\right)\right\}$ will be pseudo-convex open covering. Because $X$ is the closed $Q$ manifold it will be compact it fallows that $\left\{O_{\alpha}\right\}$ contains finite pseudo- convex sub covering.

Consider the nerve of this finite pseudo convex covering $N\left(\left\{O_{\alpha}\right\}\right)$ it is clear the geometric realization of this nerve homotopy equivalent to manifold $X$.

6) The $M^{n} \quad n$ - dimensional compact topological manifold [5], then $M^{n} \times I^{\aleph_{0}}$ is compact $Q$ - manifold [1], then as above, $M^{n} \times I^{\aleph_{0}} \times[0,1)$ will open subset in $I^{\aleph_{0}}$. The manifold $M^{n} \times I^{\aleph_{0}}$ is deformation retract in $M^{n} \times I^{\aleph_{0}} \times[0,1)$, because the manifold $M^{n} \times I^{\aleph_{0}}$ has finite pseudo- convex covering. In its turn the manifold $M^{n}$ is also deformation retract in $M \times I^{\aleph_{0}} \times[0,1)$ It follows the manifold $M^{n}$ has finite pseudo- convex covering and nerve of this covering homotopy equivalent to $M^{n}$.

7) Let $H$ local convex vector space [4] on the field real numbers $R$. It is clear that every open subset in this space has a pseudo- convex covering. Consider subset $X=\overline{\operatorname{int} X}$ in this space. Let $v \in H$ and $[0,1) \subset R$. For every $k \in[0,1)$ we have the set $k v+X$. The set $\bigcup_{k \in[0,1)} k v+X \quad$ will be open in the space $H$ it is clear that the set $X$ is deformation retract of $\bigcup_{k \in[0,1)} k v+X$. The set $\bigcup_{k \in[0,1)} k v+X$ has a pseudo- convex covering, similarly to previous cases the set $X$ has a pseudoconvex covering.

So, we see that the above class of spaces for which entropy is defined is quite wide.

It is clear that if $\left\{O_{\alpha}\right\}$ is finite pseudo convex- covering of compact topological space $X$, then geometric realization of the nerve $N\left(\left\{O_{\alpha}\right\}\right)$ homotopy equivalent to $X$

\section{Evolution of Phase Space of Closed Dynamical Systems}

Let $X_{1}, X_{2}, X_{3}, \ldots, X_{i}, \ldots$ the discrete sequence of topological spaces that describe the evolution of phase space of a some dynamical system in discrete time and let $X_{i}, i=1,2, \ldots$ compact topological spaces which admits pseudo-convex coverings. If a system is closed, then with the evolution of such a system, the entropy grows. Therefore

$$
\begin{gathered}
H\left(X_{1}\right) \leq H\left(X_{2}\right) \leq \ldots \leq H\left(X_{k}\right) \leq \ldots \\
\frac{n_{1}}{m_{1}} \leq \frac{n_{2}}{m_{2}} \leq \ldots \leq \frac{n_{n}}{m_{k}} \leq \ldots
\end{gathered}
$$

Since the desire for infinity of entropy is due to the desire for infinity $n_{i}$ we will admit that $n_{i}<n_{i+1}$ '.

During evolution in discrete time the phase space of dynamical system $X_{i}$ changing by $X_{i+1}$ We assume that $X_{i}$ and $X_{i+1}$ are different and topological spaces and $\left|n_{i+1}-h_{i}\right|<n-n_{i} \mid$, where $n$ number of elements in minimal pseudo- convex covering all other compact topological spaces $X$, which have open pseudo -convex covering. Let $\left\{Y_{J}\right\} \quad$ set of topological spaces which minimal open 
pseudo- convex covering contains $n_{i+1}$ element and $H\left(Y_{J}\right)$ is it's entropy. $H\left(X_{i+1}\right) \geq H\left(X_{i}\right)$, among $Y_{J}, j=1,2, \ldots, k$ we well choice such $Y_{j_{l}}, l=1,2, \ldots, q$ that $H\left(Y_{j_{l}}\right) \geq H\left(X_{i}\right)$. It is essential if we assume that the probability of equality $X_{i+1}=Y_{j_{l}}$ is $p_{l}=\frac{H\left(Y_{j_{l}}\right)}{\sum_{l=1}^{q} H\left(Y_{j_{l}}\right)}$. From these reasoning follows that the most probable topological space in the role $X_{i+1}$ is the space the entropy of which is closer to the number $\sum_{l}^{q} p_{l} H\left(Y_{j_{l}}\right)$.

So, as the sequence $X_{1}, X_{2}, X_{3}, \ldots, X_{n}, \ldots$ describes evolution of a phase space of closed dynamic system to the accuracy of equality of entropy.

Let new as above $n_{i} \quad i=1,2, \ldots$ number of elements of minimal pseudo convex covering of some topological space which has such covering. Let spaces $X_{i}^{\alpha_{i}}$ such that minimal pseudo-convex covering contains $n_{i}$ elements accordingly, $i=1,2, \ldots$. We denote the set of this spaces so $\mathfrak{I}_{i}$

Easy to guess that spaces from this set have finite number of spaces with different entropy. Let $\Re_{i}$, the set of classes of the spaces in $\mathfrak{I}_{i}, i=1,2, \ldots$ with equality value of entropy.

We construct a random process with discrete time $[2,13]$ in this way

Let sequence $\mathfrak{R}_{1}, \Re_{2}, \Re_{3}, \ldots \Re_{i}, \ldots$ sets of classes of spaces whit equality entropy and $q_{i}$ umber of classes $\mathfrak{R}_{i}$ $i=1,2, \ldots, i \ldots$.

Let

$$
H\left(\left[X_{i}^{\alpha_{i}}\right]_{l}\right)=H\left(X_{i}^{\alpha_{i}}\right), X_{i}^{\alpha_{i}} \in\left[X_{i}^{\alpha_{i}}\right]_{l} \in \Re, \quad l=1,2, \ldots q_{i} .
$$

We will consider class $\left[X_{i}^{\alpha_{i}}\right]_{l}$ in, $\mathfrak{R}_{i}$, let classes $\left[X_{i+1}^{\alpha_{i+1}}\right]_{j_{k}}, k=1,2, \ldots, q_{i+1}^{l}$ have entropy more than fixed class $\left[X_{i}^{\alpha_{i}}\right]_{l}$ We consider number:

$$
p_{j_{k}}=\frac{H\left(\left[X_{i+1}^{\alpha_{i+1}}\right]_{j_{k}}\right)}{\sum_{k=1}^{q_{i+1}^{l}} H\left(\left[X_{i+1}^{\alpha_{i+1}}\right]_{j_{k}}\right)}
$$

$$
\sum_{k=1}^{q_{i+1}^{l}} p_{j_{k}}=1, p_{j_{k}} \geq 0 \quad \text {. We consider matrix }
$$
$P_{i}=\left(p_{l j}^{i}\right)_{l=1,2, \ldots, q_{i}, j=1,2, \ldots, q_{i+1}}$, where $p_{l j}^{i}=p_{j_{k}}$, when $j=j_{k}$ $k=1,2, \ldots, q_{i+1}^{l}$ and $p_{l j}^{i}=0$ when

$$
H\left(\left[X_{i+1}^{\alpha_{i+1}}\right]_{j}\right) \leq H\left(\left[X_{i}^{\alpha_{i}}\right]_{l}\right), \text { le } j \neq j_{k}, k=1,2, \ldots, q_{i+1}^{l}
$$

This matrix is the transition matrix for transition random objects (classes) set $\Re_{i}$ to random objects set $\Re_{i+1}$.

In this way we have built the random process $[2,13]$ with discrete time which describe evolution phase space of closed dynamic system.

If we do not admit that the entropy of phase space of a dynamical system does not always increases, then

$$
\mathfrak{R}_{1}, \mathfrak{R}_{2}, \Re_{3}, \ldots \Re_{i}, \ldots
$$

sequence we will consider as Markov chine [2, 13] with transition matrix $P_{i}=\left(p_{l j}^{i}\right)_{l=1,2, \ldots, q_{i}, j=1,2, \ldots, q_{i+1}}$ where

$$
p_{l j}^{i}=p_{l j}^{i}=p_{j}=\frac{H\left(\left[X_{i+1}^{\alpha_{i+1}}\right]_{j}\right)}{\sum_{j=1}^{q_{i+1}} H\left(\left[X_{i}^{\alpha_{i+1}}\right]_{j}\right)}
$$

If

$$
\left[X_{1}^{\alpha_{1}}\right]^{\prime}, \quad\left[X_{2}^{\alpha_{2}}\right]^{\prime}, \ldots,\left[X_{i}^{\alpha_{i}}\right]^{\prime}, \ldots
$$

most probabilistic realization of this random process, i.e. $\left[X_{i}^{\alpha_{i}}\right]_{j}$ is the class the entropy of which is closer to the number $\sum_{j=1}^{q_{i+1}} p_{j} H\left(\left[X_{i+1}^{\alpha_{i}}\right]_{j}\right)$, then

$$
H\left(\left[X_{1}^{\alpha_{1}}\right]^{\prime}\right) \leq H\left(\left[X_{2}^{\alpha_{2}}\right]^{\prime}\right) \leq \ldots \leq H\left(\left[X_{i}^{\alpha_{i}}\right]^{\prime} \leq \ldots\right.
$$

Because in the set $\Re_{i}$ there exist the class of $n_{i}-2$ dimensional sphere $S^{n_{i}-2}$ which is most symmetric than other spaces in this set, $H\left(S^{n_{i}-2}\right)>H\left(S^{n_{i-1}-2}\right)$ and classes of spaces which minimal pseudo convex covering contains, $n_{i}$ element, which, as we known, has homotopy type of geometric realization of nerve of this covering. The lasses of every geometric simplicial complex [5] with $n_{i}$ vertex also is included in set $\mathfrak{R}_{i}$.

If we choose element from each class we will get sequence

$$
\begin{gathered}
X_{1}^{\alpha_{1}^{\prime}}, X_{2}^{\alpha_{2}^{\prime}}, \ldots, X_{i}^{\alpha_{i}^{\prime}}, \ldots \\
H\left(X_{1}^{\alpha_{1}}\right) \leq H\left(X_{2}^{\alpha_{2}}\right) \leq \ldots \leq H\left(X_{i}^{\alpha_{i}}\right) \leq \ldots
\end{gathered}
$$

So as the sequence $X_{1}^{\alpha_{1}^{\prime}}, X_{2}^{\alpha_{2}^{\prime}}, \ldots, X_{i}^{\alpha_{i}^{\prime}}, \ldots$ most probabilistic realization of the evolution of a phase space of closed dynamic system to the accuracy of equality of entropy.

Consider the $\mathfrak{R}_{i}$ set on this set we have defined probability measure $p_{i}$ on this set with way: assign to each class $\left[X_{i}^{\alpha_{i}}\right]_{j}$ the frequency:

$$
p_{i}\left(\left[X_{i}^{\alpha_{i}}\right]_{j}\right)=p_{j}^{i}=\frac{H\left(\left[X_{i}^{\alpha_{i}}\right]_{j}\right)}{\sum_{j=1}^{q_{i}} H\left(\left[X_{i}^{\alpha_{i}}\right]_{j}\right)}
$$


We can consider the random variable

$$
h_{i}: \Re_{i} \rightarrow\left\{H\left[X_{i}^{\alpha_{i}}\right]_{j}\right\}, h_{i}\left(\left[X_{i}^{\alpha_{i}}\right]_{j}\right)=H\left[X_{i}^{\alpha_{i}}\right]_{j}
$$

$$
H\left[X_{i}^{\alpha_{i}}\right]_{j} p_{i}^{j}
$$

with distribution

The mathematical expectation of this random quantity will be

$$
M\left(h_{i}\right)=\sum_{j=1}^{q_{i}} p\left(\left[X_{i}^{\alpha_{i}}\right]_{j}\right)=\sum_{j=1}^{q_{i}} \frac{H\left(\left[X_{i}^{\alpha_{i}}\right]_{j}\right)}{\sum_{l}^{q_{i}} H\left(\left[X_{i}^{\alpha_{i}}\right]_{l}\right)} H\left(\left[X_{i}^{\alpha_{i}}\right]_{j}\right)=\sum_{j=1}^{q_{i}} \frac{H\left(\left[X_{i}^{\alpha_{i}}\right]_{j}\right)^{2}}{\sum_{l}^{q_{i}} H\left(\left[X_{i}^{\alpha_{i}}\right]_{l}\right)} .
$$

\section{The Infinity Product of Probability Measures and Most Probabilistic Trajectories}

In this section we consider the question in another aspect. Consider the direct product of sets $\underset{i \in N}{\otimes} \mathfrak{R}_{i}$, on this set we have the measure $\underset{i \in N}{\otimes p_{i}}$. This measure is constructed so [3, 6, 7]: on each set we $\mathfrak{R}_{i}$ have measure defined by frequency, this measure is defined on algebra $\mathrm{A}\left(\mathfrak{R}_{i}\right)$ of subsets of $\mathfrak{R}_{i}$.

Consider the family of probability measures $\left\{\left(\Re_{i}, \mathrm{~A}\left(\Re_{i}\right), p_{i}\right)\right\}_{i \in N}$,

In the product set $\mathfrak{R}=\prod_{i \in N} \Re_{i}$ sets $A=\prod_{i \in} A_{i}$, where for finite number of $i$

$A_{i} \neq \mathfrak{R}_{i}$, these sets are called cylindrical sets, they make up a semi algebra of sets $[11,12]$, we denote this semi algebra by $\mathrm{B}(\Re)$ and smallest $\sigma$-algebra generated by $\mathrm{B}(\Re)$ so $\sigma(\mathrm{B}(\Re))$.

Let $p: \mathrm{B}(\Re) \rightarrow[0,1]$ defined so [3]:

Consider such family $\left\{A_{s_{k}}^{k}\right\}$ of elements in $\mathrm{B}(\Re)$ which have a structure:

$$
\begin{aligned}
& A_{s_{k}}^{k}=\prod_{i \in N} A_{i}, \quad A_{1}=\left[X_{1}^{\alpha_{1}}\right]_{j_{l_{1}}}, A_{2}=\left[X_{2}^{\alpha_{2}}\right]_{j_{l_{2}}}, \ldots, A_{i_{k}}=\left[X_{i_{k}}^{\alpha_{i_{k}}}\right]_{j_{l_{k}}}, A_{i_{k}+1}=\Re_{i_{k}+1}, A_{i_{k}+2}=\Re_{i_{k}+2}, \ldots \\
& k \in N, s=\left(j_{l_{1}}, j_{l_{2}}, \ldots, j_{l_{k}}\right), j_{l_{k}}=1,2, \ldots, q_{i_{k}} .
\end{aligned}
$$

Where sets $A_{1}=\left[X_{1}^{\alpha_{1}}\right]_{j_{l_{1}}}, A_{2}=\left[X_{2}^{\alpha_{2}}\right]_{j_{l_{2}}}, \ldots, A_{i_{k}}=\left[X_{i_{k}}^{\alpha_{i_{k}}}\right]_{j_{l_{k}}}$ consist of one element.

If $k$ is fixed then the family $\left\{A_{s_{k}}^{k}\right\}$ contains pairwise disjoint elements, is finite and generates probability space $\left(\left\{A_{s_{k}}^{k}\right\}, \mathrm{A}\left(\left\{A_{s_{k}}^{k}\right)\right), p\right), p\left(A_{s_{k}}^{k}\right)=p_{j_{l_{1}}}^{1} p_{j_{l_{2}}}^{2} \cdot \ldots \cdot p_{j_{l_{k}}}^{i_{k}}$

$$
\sum_{s_{k}} p_{j_{l_{1}}}^{1} p_{j_{l_{2}}}^{2} \cdot \ldots \cdot p_{j_{l_{k}}}^{k}=\sum_{j_{l_{1}}} \sum_{j_{l_{2}}} \ldots \sum_{j_{l_{k}}} p_{j_{l_{1}}}^{1} p_{j_{l_{2}}}^{2} \cdot \ldots \cdot p_{j_{l_{k}}}^{k}=1
$$

The discrete trajectory $\left[X_{1}^{\alpha_{1}}\right],\left[X_{2}^{\alpha_{2}}\right], \ldots,\left[X_{i_{k}}^{\alpha_{i_{k}}}\right]$ of the changes of the phase spaces of the system until discrete time moment $i_{k}$ we can will represent by elements of set $\left\{A_{s}^{k}\right\}$.

Consider the random vaeiable

$$
h^{k}:\left\{A_{s_{k}}^{k}\right\} \rightarrow\left\{H\left[X_{1}^{\alpha_{1}}\right]_{j_{l_{1}}} \cdot H\left[X_{2}^{\alpha_{2}}\right]_{j_{l_{2}}} \cdot \ldots \cdot H\left[X_{i_{k}}^{\alpha_{i_{k}}}\right]_{j_{l_{k}}}\right\}, h_{s}^{k}\left(A_{s_{k}}^{k}\right)=H\left[X_{1}^{\alpha_{1}}\right]_{j_{l_{1}}} \cdot H\left[X_{2}^{\alpha_{2}}\right]_{j_{l_{2}}} \cdot \ldots \cdot H\left[X_{i_{k}}^{\alpha_{i_{k}}}\right]_{j_{l_{k}}}
$$

With distribution

$$
H\left[X_{1}^{\alpha_{1}}\right]_{j_{l_{1}}} \cdot H\left[X_{2}^{\alpha_{2}}\right]_{j_{l_{2}}} \cdot \ldots \cdot H\left[X_{i_{k}}^{\alpha_{i_{k}}}\right]_{j_{l_{k}}} \_p_{j_{l_{1}}}^{1} p_{j_{l_{2}}}^{2} \cdot \ldots \cdot p_{j_{l_{k}}}^{i_{k}}
$$

The mathematical expectation of this random quantity will be 


$$
M\left(h^{k}\right)=\sum_{s} p_{j_{l_{1}}}^{1} \cdot p_{j_{l_{2}}}^{2} \cdot \ldots \cdot p_{j_{l_{k}}}^{k} H\left[X_{1}^{\alpha_{1}}\right]_{j_{l_{1}}} \cdot H\left[X_{2}^{\alpha_{2}}\right]_{j_{l_{2}}} \cdot \ldots \cdot H\left[X_{i_{k}}^{\alpha_{i_{k}}}\right]_{j_{l_{k}}}=M\left(h_{1}\right) \cdot M\left(h_{2}\right) \cdot \ldots \cdot M\left(h_{i_{k}}\right)
$$

If we take the trajectory $\left[X_{1}^{\alpha_{1}}\right],\left[X_{2}^{\alpha_{2}}\right], \ldots,\left[X_{i_{k}}^{\alpha_{i k}}\right]$ with members which entropy are closest to the corresponding mathematical expectation in the product, then this trajectory will be more probable.

Consider now such family $\left\{\bar{A}_{\bar{s}_{k}}^{k}\right\}_{k \in N}$ of elements in $\mathrm{B}(\mathfrak{R})$ which have a structure:

$$
\begin{aligned}
& \bar{A}_{\bar{s}_{k}}^{k}=\prod_{i \in N} A_{i}, \quad A_{1}=\left[X_{1}^{\alpha_{1}}\right]_{j_{1}}, A_{2}=\left[X_{2}^{\alpha_{2}}\right]_{j_{l_{2}}}, \ldots, A_{i_{k-1}}=\left[X_{i_{k-1}}^{\alpha_{2}}\right]_{j_{l_{k-1}}}, A_{i_{k}}=\Re_{i_{k}} \backslash\left[X_{i_{k}}^{\alpha_{i_{k}}}\right]_{j_{l_{k}}}, A_{i_{k}+1}=\Re_{i_{k}+1}, A_{i_{k}+2}=\Re_{i_{k}+2}, \ldots, \\
& k \in N, \bar{s}=\left(j_{l_{1}}, j_{l_{2}}, \ldots, j_{l_{k}}\right), j_{l_{k}}=1,2, \ldots, q_{i_{k}} .
\end{aligned}
$$

$k$ is not fixed. The subfamily $\left\{\bar{A}_{\bar{s}_{k}}^{k}\right\}_{k \in N} \subset\left\{\bar{A}_{s_{k}}^{k}\right\}_{k \in N}$ where $\bar{s}_{k}=j_{l_{1}}, j_{l_{2}}, \ldots, j_{l_{k}}$ fixed and for all $k \bar{s}_{k+1}=j_{l_{1}}, j_{l_{2}}, \ldots, j_{l_{k}}, j_{l_{k+1}}$. Elements of this subfamily are pairwise disjoint.

$$
\begin{aligned}
& \bigcup_{k} A_{\bar{s}_{k}}^{k}=\prod_{i \in N} \Re_{i}, p\left(A_{\bar{s}_{k}}^{k}\right)=p_{j_{l_{1}}}^{1} p_{j_{l_{2}}}^{2} \cdot \ldots \cdot p_{j_{l_{k}}}^{i_{k}}, \sum_{k \in N} \sum_{\bar{s}_{k}} p\left(A_{\bar{s}_{k}}^{k}\right)=\sum_{k \in N} \sum_{\bar{s}_{k}} p_{j_{l_{1}}}^{1} p_{j_{l_{2}}}^{2} \cdot \ldots \cdot p_{j_{l_{k}}}^{k}= \\
& =\sum_{k \in N} \sum_{j_{l_{1}}} \sum_{j_{l_{2}}} \ldots \sum_{j_{l_{k}}} p_{j_{l_{1}}}^{1} p_{j_{l_{2}}}^{2} \cdot \ldots \cdot p_{j_{l_{k}}}^{k}=1
\end{aligned}
$$

We have $\left(\left\{\bar{A}_{\bar{s}_{k}}^{k}\right\}_{k \in N}, \mathrm{~A}\left(\left\{\bar{A}_{\bar{s}_{k}}^{k}\right\}\right), p\right)$ probability space $p\left(\bar{A}_{\bar{s}_{k}}^{k}\right)=p_{j_{l_{1}}}^{1} p_{j_{l_{2}}}^{2} \cdot \ldots \cdot p_{j_{l_{k}}}^{i_{k}}$. Consider random quantity on this space:

$$
\begin{aligned}
& h_{\bar{s}}:\left\{\bar{A}_{\bar{s}_{k}}^{k}\right\} \rightarrow\left\{H\left[X_{1}^{\alpha_{1}}\right]_{j_{l_{1}}} \cdot H\left[X_{2}^{\alpha_{2}}\right]_{j_{l_{2}}} \cdot \ldots \cdot H\left[X_{i_{k}}^{\alpha_{i k}}\right]_{j_{l_{k}}}\right\}_{k \in N}, h_{\bar{s}_{k}}\left(\bar{A}_{\bar{s}_{k}}^{k}\right)= \\
& =H\left[X_{1}^{\alpha_{1}}\right]_{j_{l_{1}}} \cdot H\left[X_{2}^{\alpha_{2}}\right]_{j_{l_{2}}} \cdot \ldots \cdot H\left[X_{i_{i_{k}}}^{\alpha_{i_{k}}}\right]_{j_{l_{k}}}, \text { if } k \neq 1 \text { and } h_{\bar{s}_{k}}\left(\bar{A}_{\bar{s}_{k}}^{k}\right)=1 \text { if } k=1
\end{aligned}
$$

With distribution

$$
H\left[X_{1}^{\alpha_{1}}\right]_{j_{1}} \cdot H\left[X_{2}^{\alpha_{2}}\right]_{j_{2}} \cdot \ldots \cdot H\left[X_{i_{k}}^{\alpha_{i_{k}}}\right]_{j_{k}} \longleftarrow p_{j_{l_{1}}}^{1} p_{j_{l_{2}}}^{2} \cdot \ldots \cdot p_{j_{l_{k}}}^{i_{k}} .
$$

The mathematical expectation of this random variable will be

$$
M\left(h_{\bar{s}}\right)=\sum_{k \in N} p_{j_{l_{1}}}^{1} \cdot p_{j_{l_{2}}}^{2} \cdot \ldots \cdot p_{j_{l_{k}}}^{k} H\left[X_{1}^{\alpha_{1}}\right]_{j_{l_{1}}} \cdot H\left[X_{2}^{\alpha_{2}}\right]_{j_{l_{2}}} \cdot \ldots \cdot H\left[X_{i_{k}}^{\alpha_{i_{k}}}\right]_{j_{l_{k}}}
$$

The product $H\left[X_{1}^{\alpha_{1}}\right]_{j_{1}} \cdot H\left[X_{2}^{\alpha_{2}}\right]_{j_{2}} \cdot \ldots \cdot H\left[X_{i_{k}}^{\alpha_{i k}}\right]_{j_{k}}$ increases when increases entropy wit increasing $k$ and The product $p_{j_{l_{1}}}^{1} p_{j_{l_{2}}}^{2} \cdot \ldots \cdot p_{j_{l_{k}}}^{i_{k}}$ decreases when increase $k$, therefore, the sum will converge in many cases to a finite non-zero value.

We call this number $M\left(h_{\bar{s}}\right)$ the entropy of trajectory

$$
\left[X_{1}^{\alpha_{1}}\right]_{j_{1}},\left[X_{2}^{\alpha_{2}}\right]_{j_{l}}, \ldots,\left[X_{i_{k}}^{\alpha_{i k}}\right]_{j_{l_{k}}}, \ldots
$$

The family of $\left\{\left\{\bar{A}_{s_{k}^{\beta}}^{k}\right\}_{\beta}\right\}_{k \in N}$ contains only pairwais disjoint elements $\bigcup_{\beta}\left\{\left\{\bar{A}_{\bar{s}_{k}^{\beta}}^{k}\right\}_{\beta}\right\}_{k \in N}=\Re$, where $\beta$ varies in a countable set.

In the probability space $\left(\left\{\left\{\bar{A}_{\bar{S}_{k}^{\beta}}^{k}\right\}_{\beta}\right\}_{k \in N}, \mathrm{~A}\left(\left\{\left\{\bar{A}_{\bar{S}_{k} \beta}^{k}\right\}_{\beta}\right\}\right), p\right)$ we consider random variable on this space: $h:\left\{\left\{\bar{A}_{s_{k}^{\beta}}^{k}\right\}_{\beta}\right\} \rightarrow\left\{M\left(h_{s^{\beta}}\right)\right\}_{\beta}$ with distribution:

$$
M\left(h_{s^{\beta}}\right) \_p\left(\left\{A_{s_{k}^{\beta}}^{k}\right\}\right) .
$$

Among all possible trajectories, the most probable will be a trajectory whose entropy is closer to than all the others.

\section{Results}

1. The concept of entropy for topological spaces is defined, which admits a pseudo-convex open covering.

2 . The examples of topological spaces admitting a pseudoconvex covering are built.

3. The random process is built that describes the evolution of the phase space of a closed dynamical system up to an accuracy of equality of entropy.

4. Introduced the concept of entropy of the trajectory changes of the phase space of the system.

5. Based on the concept of entropy of trajectory the most probable trajectory of the changes phase space of a closed system is found. 


\section{References}

[1] T. A Chapmen Lecture on Q- manifolds Moscow 1981 (in Russian).

[2] J. L Doob Stochastic processes New- York -John Wiley\& Sons, London- Chapman\& Hall 1953.

[3] J. Bell Infinite product measures. Departments of Mathematics, university of Toronto, May 10, 2015.

[4] H. H. Schaefer topological vector spaces, Springer 2012.

[5] Edwin H. Spanier Algebraic Topology, MeGRAWHILL BOOK COMPANY 1966.

[6] V. I. Bogachev, Measure Theory Springer 2007.

[7] R. Ash Probability and measure theory, Academic Press; 2 edition 1999.

[8] Ya. G. Sinai, "On the Notion of Entropy of a Dynamical System," Doklady of Russian Academy of Sciences, 1959.

[9] T. Downarowicz Entropy in dynamical Systems, New

Mathematical Monograph, Cambridge University Press, Cambridge 2011.

[10] Dou Dou, Wen Huang, Kyewon Koh Park Entropy dimension of measure preserving systems cornell university arXiv: 1312.7225v2 [math. DS] 3 Jan 2014.

[11] Sadahiro SaekA Proof of the Existence of Infinite Product Probability Measures, The American Mathematical Monthly, Vol. 103, No. 8, 1996.

[12] P. BILLINGSLEY Probability and Measure, Third Edition The Universityof Chicago, A Wiley-Interscience Publication WILEY \& JOHN SONS New York, Chichester, Brisbane, Toronto, Singapore 1995.

[13] E. B. Dynkin and A. A. Yushkevich Markov Processes, English ed. Plenum Press, New York, 1969.

[14] Ryszard Engelking General topology Heldermann Verlag, 1989.

[15] Lei ZHANG Leijun LIU Wen LI Sparse Trajectory Prediction Method Based on Entropy Estimation IEICE TRANSACTIONS on Information and Systems Vol. E99-D No. 6. 2016. 